\title{
Metafilmes: La ficción audiovisual como denuncia y mitología del medio cinematográfico
}

\author{
Metafilmes: The audiovisual fiction as a mythology and \\ denunciation of the film media
}

Oscar Garaycochea

Universidad de Chile

ogaraycochea@hotmail.com

\section{Resumen}

El cine de ficción exhibe ante su audiencia pocas veces el proceso de producción que da forma a su discurso. Bastante más probable es que enrede o borre cuidadosamente las huellas de lo sucedido, para inducir la "voluntaria suspensión de la incredulidad”, un acuerdo entre los emisores y destinatarios del discurso, del que dependería cualquier disfrute satisfactorio de una ficción. Para la industria del cine resulta más productivo elaborar una mitología encubridora del medio, que utilizarlo para denunciar su funcionamiento. La primera actitud predominó durante el medio siglo que se prolongó el sistema de los grandes estudios. La segunda actitud, denunciadora, crítica o simplemente narcisista, se manifestó en el siguiente medio siglo, centrándose algunas veces en aspectos biográficos del proceso de producción o en una crítica del sistema de representación del medio.

Palabras clave: Metalenguaje, metafilmes, cine en el cine

\begin{abstract}
The fictional cinema rarely exhibit to its audience the production process that gives shape to its discourse. But quite more probable is that carefully get mixed or deleted the traces of what happened, to induce the "voluntary suspension of disbelief", an agreement between producers and audiences of the discourse, of wich depends any satisfacory enjoyment of a fiction. For the cinema industry is more productive to elaborate a mythology covering of the media, rather than to use it to report its operation. The first attitude prevailed during the half century that big studios system lasted. The second attitude, a reported, critic or just narcissistic one, manifested during the next half century, focusing sometimes on biographical aspects of the production process or on media representation system's critic.
\end{abstract}

Keywords: Metalanguage, Metafilmes, Movies at the movies 


\section{Eclipse y autorreferencia de la producción audiovisual}

Aquellos que hacen cine lo hacen de forma colectiva. Su participación suele confundirse con la de muchos otros. Pocos son aquellos que consiguen ser identificados. Los actores son los más ostensibles, porque sus nombres ocupan la pantalla, gozan de créditos aislados y suelen protagonizar el discurso de la prensa de espectáculo. Comparado con los actores, un guionista, director o fotógrafo debe resignarse a que su trabajo tal vez no pase desapercibido, pero de todos modos serán muy pocos quienes se los atribuyan. A pesar de que algunos analistas celebran el carácter colectivo del cine (cf. Hauser, 1985), un punto de vista compartido por algunos artistas (cf. Bergman, 1997), otros confían utilizar el medio para referirse a su entorno, revelar sus conflictos ante la cámara, en lugar de permanecer detrás.

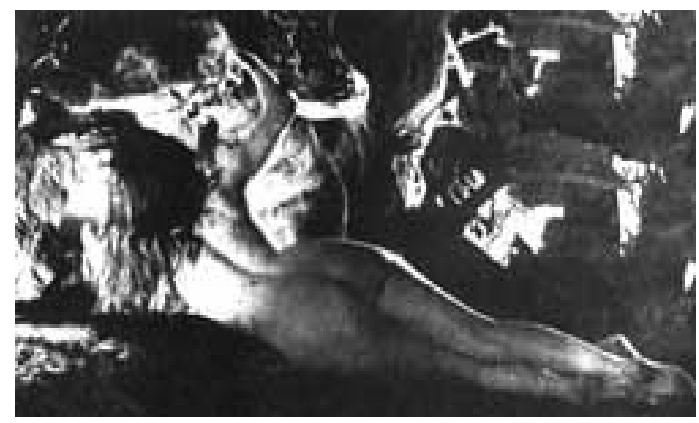

Figura 1

No es raro que un artista de cualquier disciplina se refiera a su oficio, a la gente que lo rodea, a sus preocupaciones más personales, porque se trata de las circunstancias que conoce mejor. La pintura de Picasso adopta durante los últimos años del artista la relación que se da entre el pintor y su modelo como tema fundamental. En otros casos puede incluirse la relación entre el pintor y sus colegas (como se observa en la visión del taller del artista de Gustave Courbet). En el teatro, hay piezas que muestran el funcionamiento de un equipo de producción (Hamlet de William Shakespeare, Il teatro Cómico de Carlo Goldoni, Sei Personaggi in cerca d'Autore de Luigi Pirandello o Living in Oblivion de Tom DiCillo). En el ámbito de la literatura, hay novelas que revelan el proceso de creación de una historia (Paludes de André Gide o 62, Modelo para armar de Julio Cortázar). De acuerdo a la definición clásica de Roman Jakobson, la función metalingüística o de glosa, es aquella donde el discurso se centra en el código (cf. Jakobson, 1981: 358).

La posibilidad de exhibir ante la audiencia el proceso de producción de la obra de arte, es una tentación que han sentido los artistas de todos los tiempos, una situación que en la modernidad ha pasado a convertirse en uno de los temas recurrentes. ¿Por qué centrarse en la rutina del artista? Primero, porque ofrece un ambiente pródigo en conflictos, que habitualmente suele escamotearse a la audiencia que admira el producto concluido. Luego, porque permite a 
los artistas exhibirse como personajes tan atormentados y dignos de piedad como los héroes clásicos.

Charles Chaplin fue uno de los primeros en incluir la rutina de los estudios de Mack Sennet como tema de sus comedias. En A film Johnnie (1914) aparece como un aficionado que aspira a introducirse en la nueva industria (una situación que se encontraba muy próxima a la de Chaplin). En The Property Man (1914) regresa al mismo lugar y se concentra en el trabajo del utilero. His new Job (1915), fig. 1, revela el sistema de varias filmaciones simultáneas en un mismo estudio y las confusiones cómicas que surgen del tránsito de una a otra, un recurso que seis décadas más tarde utilizó Mel Brooks en Blazzing Saddles (1974).

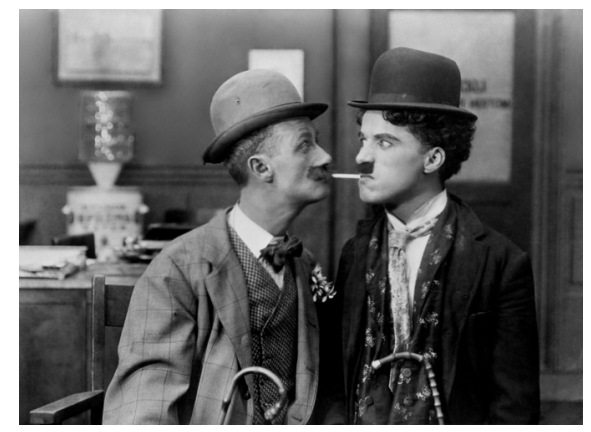

Figura 2

Haxan (1922), fig. 2, es un filme de ficción que recrea circunstancias históricas de la persecución a las brujas en Dinamarca, durante el siglo XVI, que de vez en cuando se interrumpe para incluir escenas didácticas sobre las técnicas de tortura, o entrevistas a los actores que se presentan como tales. La heterogeneidad del discurso multiplica las fracturas en el proceso de recepción, obligando a la audiencia a interrogarse constantemente sobre su actitud respecto de la información suministrada por la pantalla. El efecto es similar al del cine de Godard y Gorin, medio siglo más tarde, en Rose et Vladimir (1971), cuando cuestionaban la ideología predominante en el espectáculo fílmico, mediante una pareja de montajistas que discute el sentido de las escenas que trabajan. La audiencia es obligada a involucrarse en el contradictorio armado de la ficción y a diferenciar las partes que la componen y no terminan de soldarse.

El cine clásico, producido en la primera mitad del siglo xx, prefiere mantener una atmósfera de malentendidos y silencio sobre sus procesos productivos. Si la industria de Hollywood era el escenario de Goldwyn Follies (1938) y las dos primeras versiones de A Star is Born (1937): la dirigida por William Wellman -fig. 3- así como en la de George Cukor (1954), no es mucha la información que suministran sobre la elaboración de filmes, porque el trabajo de los actores que protagonizan esas historias apenas se muestra. 
Ellos firman contratos, asisten a estrenos y reciben premios, sufren depresiones, pero nunca ensayan, nunca se equivocan. La materialidad de la producción audiovisual es escamoteada. Hollywood es visto como un lugar excepcional, en el que se vuelven posibles rápidos cambios de fortuna y cualquiera puede pasar del anonimato a la fama (y viceversa) en muy poco tiempo y sin que se entienda muy bien por qué sucede. Es la perspectiva mitológica de la prensa de espectáculos, cuyo discurso elude cualquier dato que ponga en riesgo la imagen oficial del medio. En él, los actores y productores son quienes más se destacan y mitifican. Unos porque ocupan el encuadre y la publicidad con sus presencias seductoras, los otros porque ostentan su marca en todo lo que ofrecen.

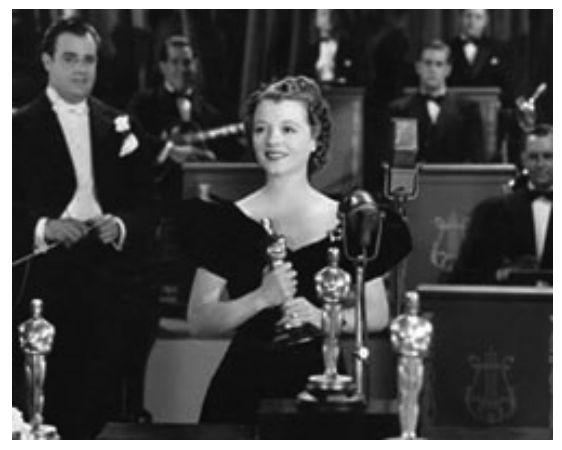

Figura 3

La técnica de los actores nunca se analiza, como tampoco la ética, ni los negocios de los productores. Ellos no parecen tener fallas. Cuando los actores quedan fuera del medio, es por sus debilidades personales (como el alcoholismo del protagonista masculino de A Star is born), una situación que el discurso presenta como desconectada del entorno laboral.

El productor de Goldwin Follies es un hombre maduro, paternal, que voluntariamente sale en busca del destinatario ideal de sus productos, para convertirlo en guía de sus decisiones, hasta que lo halla en una joven pueblerina, defensora de los valores más tradicionales de su comunidad. ¿Cómo expresar mejor el mito de una industria puesta al servicio del consumidor?

\section{El productor audiovisual como demiurgo}

Tanto en la comedia Sullivan's Travels (1941) como en The Majestic (2000), la industria del cine que se estableció en Hollywood en la segunda década del siglo XX, aparece como un ambiente tan opuesto a la creatividad individual, que los personajes protagónicos deben huir de él, para evitar que los aplaste. En el filme de Sullivan's Travels, el Hollywood de los cinco grandes estudios, dueños también de cadenas de distribución, contamina todo lo que toca y aquellos que disfrutaron la experiencia de participar en la industria, no pueden quitarse la marca. En The Majestic, ambientada a comienzos de los 50, durante la caza de brujas del senador McCarthy, la 
industria ha pactado con el extremismo político y no duda en destruir a sus colaboradores con tal de mantener intactas las bases del negocio.

Algo distinto ocurre en The Bad and the Beautiful (1952), que revela el poder omnímodo del productor. Ajeno a toda preocupación artística, creativo a su manera pero también destructor de quienes se encuentran sometidos a sus iniciativas, el productor infecta y fecunda al mismo tiempo. La industria no existiría sin él, aunque el arte suele ser su primera víctima. Un escritor, una actriz y un director son manipulados por él.

Cuando el personaje regresa en Two Weeks in Another Town (1962) el panorama de la industria ha cambiado. Hollywood ha dejado de ser el centro de la mitología audiovisual, el régimen de los grandes estudios se encuentra en ruinas y los artistas que antes gozaban de estabilidad buscan en otra parte (Roma) continuar una carrera cada vez más incierta.

El director y productor de Bowfinger (1999) es un pillo que ha sido repetidamente rechazado por el sistema y tiene que arreglárselas con los desechos que obtiene por azar. Con ese material considerado inútil por todo el mundo, arma un filme de ficción en torno a un actor que ignora haber sido convertido en protagonista de un filme y considera ser víctima de un complot extraterrestre. Si bien la comedia impone reglas tales como la improbabilidad de las situaciones y el happy end, el filme informa que en Hollywood cualquier delirio puede obtener la aprobación de la audiencia, y en ese caso pasa a ser aceptado como discurso sensato.

En Il Caimano (2006), el productor es una de las víctimas de la industria audiovisual italiana, que a comienzos de los ' 80 privilegió el desarrollo de la televisión y condenó a muerte al cine. No es un superviviente nostálgico de la época de oro del cine italiano, sino un fabricante de derivados de la producción industrial (uno de sus filmes se titula Maciste contra Freud), que arrienda un pequeño estudio en el que se ha dedicado a los infomerciales. Por descuido, se involucra en nada menos que la realización de un filme de denuncia sobre el ascenso al poder de Berlusconi.

\section{El medio inaccesible}

Llegar al cine, incorporarse a la producción audiovisual, es el objetivo de muchos miembros de la audiencia. Pocos logran acceder al medio y son menos los que permanecen. El medio utiliza y desecha a la mayor parte de quienes lo producen.

En Sunset Boulevard (1950), fig. 4, hay una visión sesgada del medio, porque los personajes son marginales, en un caso debido a que perdieron vigencia muchos años antes (le sucede a la envejecida actriz del cine mudo, Norma Desmond y al director Max von Mayerling, quien veinte años antes fue su director y esposo, para convertirse luego en su mayordomo y guardián) o porque no logran incorporarse a la producción (es la situación del guionista sin trayectoria profesional, cuyos proyectos son rechazados). Sunset Boulevard es producto de uno de los cinco grandes estudios de la época (Paramount), por lo que no es de extrañar que la industria misma aparezca libre de responsabilidad en la marginación de guionista, director y actriz. 
En Singin'n the Rain (1952) los desplazados son los actores que afrontan el paso del cine mudo al sonoro. No se trata sólo de adoptar ciertas normas técnicas rudimentarias (la captación del sonido por los micrófonos de fines de los años '20) que aseguran la posibilidad de continuar en la industria, sino de la caducidad de un repertorio expresivo y la búsqueda de otro, no siempre accesible a todos los miembros del equipo productor. Hay géneros completos que entran en crisis (los melodramas de época) y géneros que prosperan (la comedia musical), tal como hay actores que se adaptan a las nuevas demandas del medio y otros que resultan marginados (la actriz de voz chillona). Como Singin'n the Rain es el filme de otro gran estudio (MGM) se impone una visión mítica del medio: el actor sería el centro del proceso productivo, tal como lo es del proceso de distribución. Pase lo que pase, la crisis del medio tiene un desenlace feliz, la empresa capitaliza las iniciativas de los artistas que mantiene empleados. Sólo quedan fuera los antagonistas, aquellos que desde la perspectiva valórica de la audiencia merecen ese castigo.

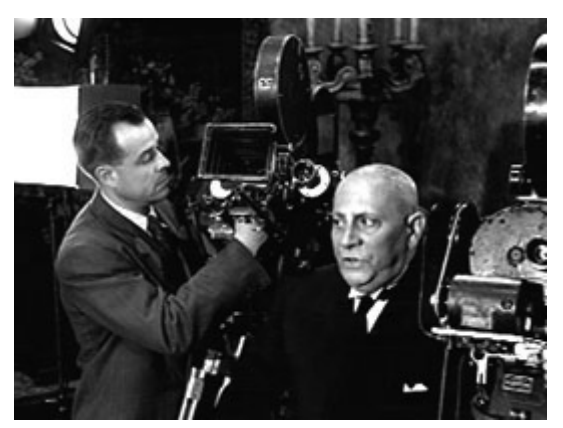

Figura 4

\section{Autorretrato del director de cine como héroe y víctima}

Hay que esperar hasta Otto e Mezo (1963) para ver un filme donde el trabajo del director del equipo audiovisual se revela como un tema atractivo para la audiencia. Lejos queda la figura del cineasta discreto, que cuenta historias ajenas y disimula las confesiones (como sucedía con el cine de John Ford o Howard Hawks). Para Fellini, lo autobiográfico es el centro, pero no debe tomarse literalmente. Si bien viste a un actor con una chalina y un sombrero idéntico a lo que él usa, elige a Marcello Mastroianni como intérprete, con lo que agrega un glamour que no se corresponde con su figura. La misma sublimación se da en la esposa del director ficticio, Anouk Aimé en lugar de la menos atractiva Giulietta Massina. Quizás los diálogos con el guionista sean parecidos a los que Fellini mantenía con Ennio Flaiano, Tullio Pinelli y Brunello Rondi, pero en el filme todos ellos son resumidos en uno. En cuanto al rol inspirador de Claudia Cardinale, ella se interpreta a sí misma y Fellini no suministra ningún dato que no sea el de su imagen pública. 


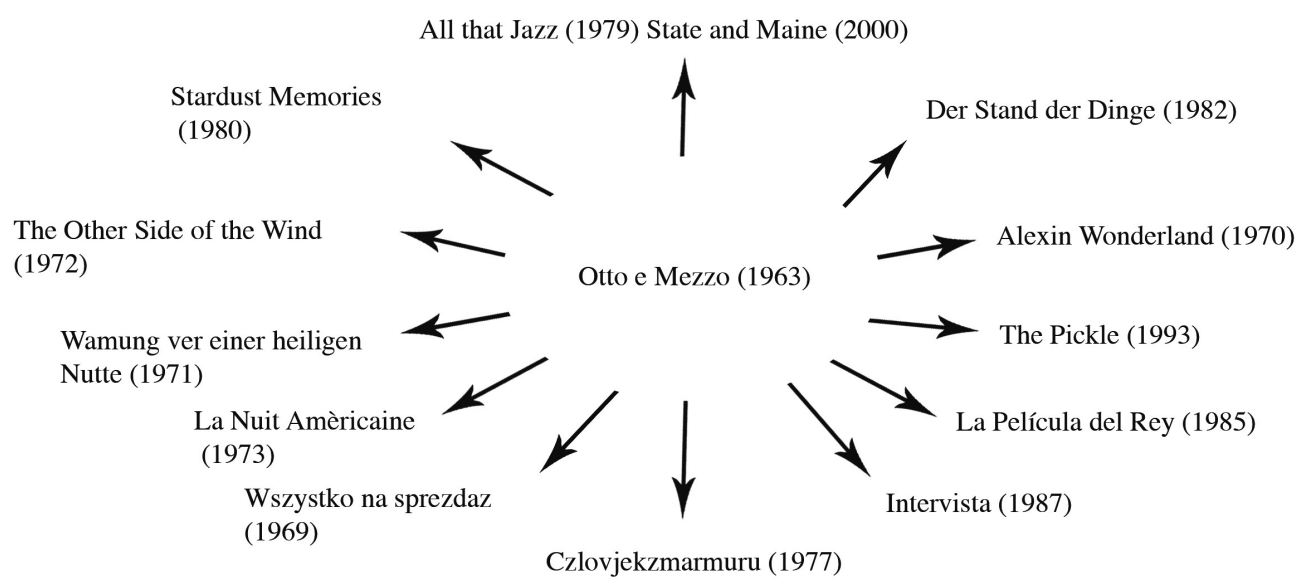

Figura 5

De la producción audiovisual, Otto e Mezo muestra los preparativos y, sobre todo, el pánico que se apodera del director y lo impulsa a desechar todo lo que había preparado. A pesar del develamiento que promete, Otto e Mezo revela lo menos posible. Antes que una visión crítica, es un strip tease que demora la desnudez y deriva en escapismo. Fellini utiliza los conflictos de la preproducción para referirse apenas al estado de ánimo del director-autor que pocos años antes asumió un rol nuevo (y mítico) de artista solitario, gracias a la crítica europea de cine que privilegió su aporte sobre el de los actores y productores.

El efecto de Otto e Mezo entre los profesionales de la producción audiovisual, fue considerable. Fellini, uno de los creadores más prestigiosos del medio, optaba por exhibirse en la pantalla, con todas sus miserias. Eso autorizaba a otros menos célebres, incluso completos desconocidos, a imitarlo sin avergonzarse, en la confianza de hallar una audiencia atenta. Desde Otto e Mezzo, las visiones parciales del trabajo de producción audiovisual no han dejado de sucederse. Cineastas de renombre no han dudado en seguir el modelo o imitarlo con un mínimo de variantes (como hicieron en distintos momentos Woody Allen, Bob Fosse, Carlos Sorin o Phil Joanou). En el caso de Fassbinder, dedica un filme a la ausencia del director en un equipo productor similar al suyo. Otros filmes han desviado el centro de atención, desde el director hacia el productor ausente (Wender) o los actores que mueren durante la producción (Wajda).

La lista de filmes que derivan de Otto e Mezo no intenta ser exhaustiva, pues debería incluir una serie de filmes iraníes recientes de Mosehn Makhmalbaf (Nun va Goldoon y Salaam Cinema), Abbas Kiarostami (Nema-je Nazdik, Zare darakhatan zeyton y Bad mara khahad bord) y Jafar Panahi (Ayneh), que develan diferentes momentos del proceso de producción 
cinematográfico y ponen frente a la cámara al mismo director o a un actor que se presenta como él, conservando sus nombres y atributos.

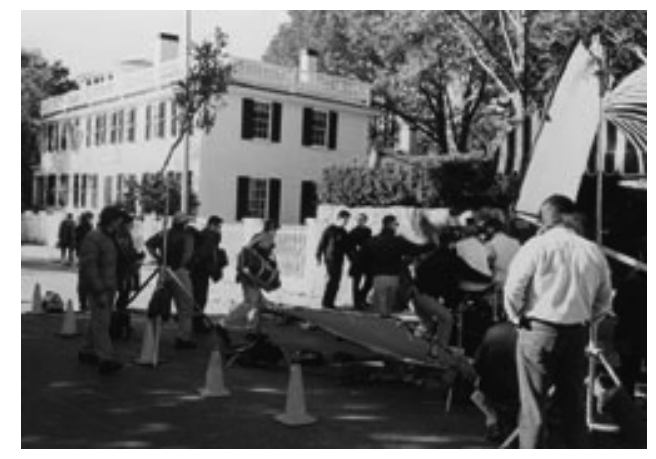

Figura 6

La figura del director adquiere en el cine iraní un carácter metafórico. Suele ser él mismo, pero el tema de los filmes es menos el poder excesivo que él detenta que sus circunstancias biográficas. El director es alguien que da órdenes que no siempre se siguen a cabalidad, pero no pueden ser discutidas por aquellos que deben seguirlo.

\section{Develamiento del medio}

En el cine de las últimas décadas, la inclusión del proceso de producción ha pasado a convertirse en uno de los rasgos fundamentales de la crítica al sistema. Durante la etapa maoísta de Jean-Luc Godard (cuando su responsabilidad de artista célebre desapareció en las obras del colectivo Dziga Vertov que formó con Jean-Pierre Gorin), filmó el western Vent d'Est (1972), con el único objeto de desmontarlo técnica e ideológicamente durante la proyección, mediante las voces de los narradores en off y los carteles intercalados.

Casi cuarenta años después de Otto e Mezzo, la posibilidad de mantener la imagen sublime del creador se ha devaluado. El director impotente de State and Main (2000), fig. 6, se encuentra más relacionado con el de La Nuit Américaine (fig. 7) que con el agónico artista de Otto e Mezzo, por el tono de comedia y el escaso énfasis que se le otorga al director, para revelar la multiplicidad de aportes contradictorios que da forma a un filme independiente. La fragilidad del trabajo creativo en todos los niveles y el peso decisivo que adquiere el fundamento mercantil del cine, son los aspectos que se destacan.

El tono de sátira se impone al drama en Adaptation (2002), filme centrado en el proceso de escritura de un guión: ¿cómo adaptar a las convenciones del cine de Hollywood la materia de un reportaje periodístico? ¿Cómo logra la retórica de un medio imponerse a cualquier material que penetre en su radio de influencia? ¿Cómo eludir los estereotipos canonizados por las master classes de Robert McKee, seguidas con fervor religioso por centenares de aspirantes a guionista? Los códigos neo-aristotélicos que rigen en Hollywood desde hace casi un siglo, 
metabolizan cualquier idea original, dejan su huella en la erotización de los conflictos, las curvas dramáticas dotadas de clímax, los desenlaces moralizantes, etc.

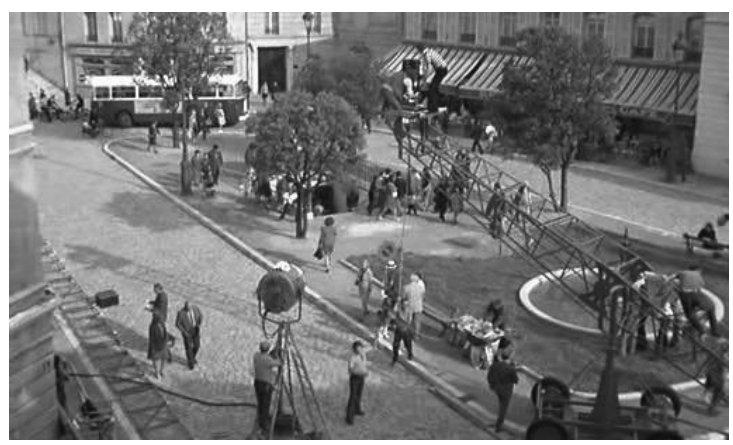

Figura 7

Junto a esos filmes debería incluirse también derivaciones que se ambientan fuera de la industria de largometrajes. En Inserts (1975) se revela la marginalidad de los cineastas dedicados a la pornografía a comienzos de los años '30. En La Tarea (1990), la búsqueda de originalidad por un estudiante de cine. En Entropy (2001), el conflicto entre la lucrativa profesión de director-productor de video-clips y la aspiración a una obra más ambiciosa.

Es interesante la obra de Nuri Bilge Ceylan, cineasta turco que muestra la elaboración de su filme Mayissi kintisi (1999), que incluye un fragmento de su vida, el decorado de su pueblo natal, los personajes de sus padres y vecinos... pero se excluye a sí mismo, reemplazándose por un actor, porque él se encuentra detrás de la cámara.

Impresiona la cantidad de artistas destacados que entre los años 60 y 70 no dudaron en utilizar este esquema célebre y, por lo tanto, fácil de reconocer para la audiencia (motivo por el cual, después de tanto uso podría estar perdiendo su valor expresivo inicial para derivar en parodia, como se advierte en Mazursky, que ha utilizado dos veces el esquema de Otto e Mezo, primero en serio (Alex in Wonderland), luego en farsa (The Pickle).

Fellini mismo rehizo su filme en Intervista (1987). En lugar de presentarse como un artista fotogénico, atormentado, personificado por un actor célebre, Fellini se presentó a sí mismo, adiposo, envejecido, carente de proyectos y convertido en el entrevistado de unos periodistas japoneses, en medio de un estudio que ya no hace cine sino publicidad, con actores que se han retirado (Anita Eckberg) o trabajan en infomerciales (Marcello Mastroianni).

\section{Conclusiones}

La reaparición del discurso metalingüístico a lo largo de la historia del cine, ha terminado por definir las reglas de un género menor, pero imposible de ignorar, que prospera en ciertos momentos de crisis de los códigos de representación dominantes en el medio: la nouvelle vague francesa y el new american cinema de los 60, el cine alemán de los 70, el cine iraní de los 90. 


\section{Referencias bibliográficas}

Bergman, I. (1997). «¿Qué es la realización cinematográfica?». Estudios Cinematográficos. № 10.

Jakobson, R. (1981). Ensayos de Lingüística General. Barcelona: Seix Barral.

Hauser, A (1985). Historia Social del Arte y la Literatura. Barcelona: Labor.

\section{Referencias filmográficas}

Allen, W., dir. y lib. (1980). Stardust Memories. Estados Unidos: Rollins-Joffe.

Belvaux, R. y Bonzel, A., dir. y lib. (1992). C'est arrivé près de chez vous. Bélgica: Les Artistes Anonymes.

Bilge Ceylan, N., dir. y lib. (1999). Mayis sikintisi. Turquía: NBC Ajans.

Brooks, M., dir.; Brooks, M. y Steinberg, N., lib. (1974). Blazzing Saddles. Estados Unidos: Warner.

Burton, T., dir.; Grey, R. y Alexander, S., lib. (1994). Ed Wood. Estados Unidos: Touchstone.

Byrum, J., dir. y lib. (1974). Inserts. Reino Unido: Film \& General Prod.

Chaplin, Ch., dir. y lib. (1915). His new Job. Estados Unidos: Essanay.

_ (1914). The Property Man. Estados Unidos: Keystone.

Christensen, B., dir. y lib. (1922). Häxan. Suecia, Dinamarca: Aljosha-Svensk Filmindustri.

Cukor, G., dir.; Hart, M. y Parker, D., lib. (1954). A Star is born. Estados Unidos: Warner.

Darabont, F., dir.; Sloane, M., lib. (2001). The Majestic. Estados Unidos, Australia: Darkwoods.

Fassbinder, R. W., dir. y lib. (1971). Warnung vor einer heiligen Nutte. Alemania: Tango-Nova-Antiteater X.

Fellini, F., dir.; Fellini, F. y Angelucci, G., lib. (1987). Internista. Italia: Aljosha-RAI-Cinecittà.

Fellini, F., dir.; Fellini, F. y Flaiano, E., lib. (1963). Otto e mezzo. Francia, Italia: Cineriz-Francinex.

Fosse, B., dir.; Fosse, B. y Arthur, R. A., lib. (1979). All that Jazz. Estados Unidos: Columbia-XXth Century Fox.

Godard, J.-L., dir.; Moravia, A., lib. (1963). Le Mépris. Francia, Italia: Champion-Concordia-Rome París.

Groupe Dziga Vertov (1971). Rose et Vladimir. Francia, Alemania: Munich Tele-Pool. (1970). Le Vent d’Est. Francia, Alemania, Italia: Anoushka-Filmkunst-Polifilm.

Hermosillo, J., dir. y lib. (1991). La Tarea. México: Clasa.

Joanou, Ph., dir. y lib. (1999). Entropy. Estados Unidos: Baldwin/Cohen-Tribeca.

Jonze, S., dir.; Kauffman, Ch., lib. (2002). Adaptation. Estados Unidos: Beverly Detroit-Intermedia.

Kiarostami, A., dir. y lib. (1999). Bad ma ra khahad bord. Irán, Francia: MK2. 
(1994). Zire darakhatan zeyton. Francia, Irán: CiBy 2000-Farabi Cinema Foundation.

(1990). Nema-je Nazdik. Irán: Instituto for Intellectual Development of Children and Young Adults.

Makhmalbaf, M., dir. (1996). Nun va Goldoon. Francia, Irán: MK2-Pakhshiran.

_ (1995). Salaam Cinema. Irán: Amoon.

Marshall, G., dir.; Hecht, B. y Kuller, S., lib. (1938). Goldwyn Follies. Estados Unidos: Samuel Goldwin.

Mamet, D., dir. y lib. (2000). State and Maine. Francia, Estados Unidos: Filmtown-Green/RenziEldorado.

Mazursky, P., dir. y lib. (1993). The Pickle. Estados Unidos.

Mazursky, P., dir.; Mazursky, P.y Tucker, L. (1970). Alex in Wonderland. Estados Unidos: Metro Goldwin Meyer.

Minelli, V., dir.; Shaw, I. y Schnee, Ch., lib. (1962). Two Weeks in another Town. Estados Unidos: Metro Goldwin Meyer.

Minelli, V., dir.; Schnee, Ch. y Bradshaw, G., lib. (1952). The Bad and the Beautiful. Estados Unidos: Metro Goldwin Meyer.

Moretti, N., dir.; Moretti, N. y Schleef, H., lib. (2006). Il Caimano. Francia, Italia: Sacher-Bac-StéphanFrance 3-Canal+.

Moretti, N., dir. y lib. (1998). Aprile. Italia, Francia: Bac-RAI-Sacher-La Sept Cinéma-Canal+.

__ (1993). Caro Diario. Italia, Francia: Banfilm-RAI-Sacher-La Sept Cinéma-Canal+.

Nichols, G., dir.; Hutchinson, C., lib. (1914). A Film Johnnie. Estados Unidos: Keystone.

Oz, F., dir.; Martin, S., lib. (1999). Bowfinger. Estados Unidos: Universal.

Panahi, J., dir. y lib. (1997). Ayneh. Irán: Rooz.

Panahi, J., dir.; Kiarostami, A., lib. (1995). Badkonake sefid. Irán: Ferdos.

Sorín, C., dir.; Sorín, C. y Goldenberg, J., lib. (1986). La película del Rey. Argentina: Motion.

Sturges, P., dir. y lib. (1941). Sullivan's Travels. Estados Unidos: Paramount.

Truffaut, F., dir.; Richard, J.-L. y Schiffmann, S., lib. (1975). La Nuit Américaine. Francia, Italia: Les Films du Carosse-PECF-PIC.

Wajda, A., dir. (1977). Czlovjek z marmuru. Polonia: Polsky-Zéspol.

_ (1969). Wszystko na sprzedaz. Polonia: Zespol «Kamera».

Welles, O., dir. y lib. (1972). The Other Side of the Wind. Francia, Irán: Les Films de l'AstrophoreSACI.

Wellman, W., dir.; Wellman, W. y Carson, R., lib. (1937). A Star is born. Estados Unidos: Selznick. 
Wenders, W., dir.; Kramer, R. y Wallace, J., lib. (1982). Der Stand der Dinge. Alemania, Estados Unidos, Portugal: Gray City-Proyect-Road Movies-ZDF.

Wilder, B., dir.; Brackett, Ch., Marshman, D. M. y Wilder, B., lib. (1950). Sunset Boulevard. Estados Unidos: Paramount. 